\title{
Research on the Low Frequency Broadband Piezoelectric-Magnetostrictive Hybrid Transducer
}

\author{
Duo Teng', and Ning $\mathrm{Zhu}^{1,2}$ \\ ${ }^{1}$ School of Marine Science and Technology, Northwestern Polytechnical University, Xi'an 710072, China \\ ${ }^{2}$ National Key Laboratory for Underwater Information Processing and Control, Xian 710072, China \\ Correspondence should be addressed to Duo Teng; tengduo@nwpu.edu.cn
}

Received 18 November 2015; Accepted 24 January 2016

Academic Editor: Francisco R. Villatoro

Copyright (c) 2016 D. Teng and N. Zhu. This is an open access article distributed under the Creative Commons Attribution License, which permits unrestricted use, distribution, and reproduction in any medium, provided the original work is properly cited.

\begin{abstract}
The effective ways for underwater transducer to lower its operating frequency, to broaden its bandwidth, and to develop its miniaturization are investigated. According to the theory of coupled multimode vibrations, a novel Hybrid transducer is developed. Different from the traditional Hybrid transducer, the improved point is the low frequency vibration controlled by the zigzag piezoelectric section and the high frequency vibration controlled by the one-dimensional magnetostrictive section. Through building the equivalent circuit model and finite element model, the performances of transducer will be predicted. The analysis shows that FEM is suitable for analyzing such a Hybrid underwater transducer within $5 \%$ deviation. The corresponding tests show that the volume and weight of the Hybrid transducer undergo a sharp drop after improvement. The novel Hybrid transducer has a distinct advantage in low frequency, bandwidth, and miniaturization. The prototype has the resonance at $1.82 \mathrm{kHz}$ and $3.76 \mathrm{kHz}$. It can be used effectively in the bandwidth of $1.5 \mathrm{kHz}$ to $5 \mathrm{kHz}$. Its main body has an external diameter of $54 \mathrm{~mm}$. The whole prototype is $235 \mathrm{~mm}$ long and weighs $2.61 \mathrm{~kg}$.
\end{abstract}

\section{Introduction}

Compared with radio waves, the acoustic wave is still the most effective way to realize the detection, navigation, and ranging in water. In this process, the underwater transducer is an indispensable electroacoustic device, which utilizes the certain physical effect to transform energy between acoustic and electrical signals [1]. Such devices have extensive applications in many fields, such as underwater detection, underwater communication, ocean exploitation, collection of scientific data recorded at the ocean-bottom stations, and remote control in offshore oil industry. In general, in order to obtain the further detection range and the more available information, the transducers with the performance of low frequency, broad bandwidth, high power, and miniaturization become the development trend [2]. Especially accompanied by the improvement of microelectronics and information processing technology, it is becoming the bottleneck problem to lower the operating frequency and to broaden the bandwidth. In the last dozens of years, some research works have realized the outstanding effect. In 2002, a triply resonant broadband transducer fabricated at the Naval Undersea Warfare Center has generated three resonances within 15 and $35 \mathrm{kHz}$ ranges [3]. In 2009, a new low frequency, broadband free-flooded piezoelectric underwater acoustic transducer researched by $\mathrm{Lu}$ et al. has achieved the bandwidth of 3 $7 \mathrm{kHz}$ within the fluctuation of $6 \mathrm{~dB}$ [4]. In 2011, a broadband flextensional transducer designed by Pan et al. has achieved the bandwidth of $2.2 \sim 9 \mathrm{kHz}$ within the fluctuation of $10 \mathrm{~dB}$ [5]. Considering the numerous achievements, multiple resonances technology is still one of the most effective ways to broaden the operating bandwidth. Here we will present a novel Hybrid transducer which can satisfy the characteristics of low frequency, broad bandwidth, and miniaturization.

\section{Piezoelectric-Magnetostrictive Hybrid Transducer}

The Hybrid transducer is a longitudinal vibrator composite bar type one with the active stacks composed of piezoelectric 


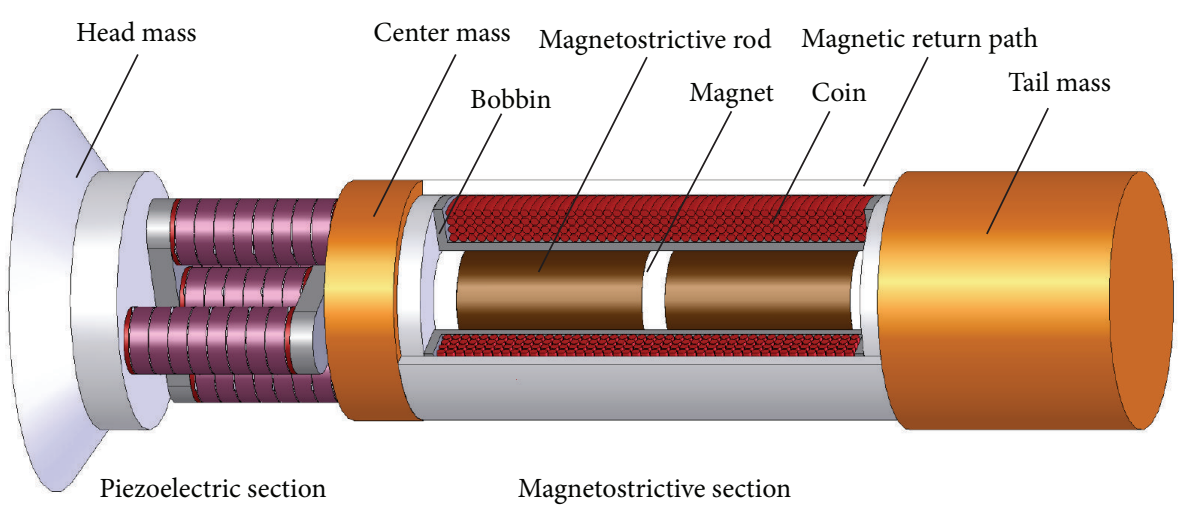

FIgURE 1: Schematic diagram of the Hybrid transducer.

ceramic and giant magnetostrictive material in mechanical series [6]. Its main components include three mass loads (i.e., head mass, center mass, and tail mass) and two active stacks (i.e., piezoelectric section and magnetostrictive section). Generally, the piezoelectric section is made of thin piezoelectric ceramic rings in series between the head mass and center mass, and the magnetostrictive one consists of the magnetostrictive rod, permanent magnet, coil, magnetic return path, and so forth between the center mass and tail mass. Both sections should be tightened by prestressed bolts. Remarkably, the Hybrid transducer can be driven in two different ways. The piezoelectric stack is always voltage excited, while the magnetostrictive one is current excited [7]. When the suitable couple between the double mode vibrations occurred, the bandwidth of the transducer will be broadened. This is the most favorable point. Also, this type transducer has some other unique properties such as selftuning, low operating frequency, and high electroacoustic energy conversion efficiency [8]. So it becomes a common source applied in the underwater field.

Figure 1 shows the schematic diagram of our Hybrid transducer, which is different from the classical one. The main distinction lies in the piezoelectric active stack. Conventionally, a single piezoelectric stack with longitudinal vibration is used, so that the piezoelectric stack can control the higher frequency, while the magnetostrictive section can control the lower frequency. As improved, a zigzag structure will be substituted for the single bar in piezoelectric section (see Figure 1). The advantage of doing so is that the piezoelectric section will control the lower frequency; at the same time the frequency controlled by the magnetostrictive section will still be kept as before without any change. The reason for the above effect lies in the zigzag structure which can give a distinct lower frequency vibration. The zigzag configuration like the folding ruler will take full advantage of the volume of space, and then the certain transform will be achieved from one to multiple times of the effective length based on the longitudinal vibration. The suitable couple between the longitudinal extensional vibration and flexural vibration of the novel configuration will be beneficial to solve the coexistence between the low frequency and miniaturization of the underwater transducers, and this is also one effective way to broaden the bandwidth and to improve the ratio of transmitting power to weight.

\section{Equivalent Circuit Model of the Hybrid Transducer}

It was Mason who first introduced the equivalent circuit model for transducers in sonar [9]. Electrical equivalent circuit is an equivalent representation using certain electrical elements instead of mechanical or acoustic parts for a complex system. For example, we can use the inductors, resistors, and capacitors to represent mass, resistance, and compliance (reciprocal of spring constant), respectively, use the voltage to represent the force, and use the current to represent the velocity $[10,11]$. Such an equivalent circuit is especially useful since transducers are always connected to electrical components. It is one of the most effective ways to design and estimate a transducer quickly and conveniently.

Before modeling our Hybrid transducer with equivalent circuit method, some assumptions have to be considered according to its structure and application as follows:

(1) Since the size of identifiable moving parts is much smaller in terms of dimensions compared to the wavelength in the material, all the flexural vibrations will not be considered, including the flexural vibration of radiating head which looks like a horn and the flexural vibration of the beams assembled in the piezoelectric section.

(2) All the prestressed bolts will not be considered.

(3) All the piezoelectric discs are glued in electrical parallel and in mechanical series. It can be derived that an axially poled thin bar will take the place of the above stack [12].

(4) Within the piezoelectric ceramic disc, consider the electric field $E_{1}=E_{2}=0$ because of the small thickness, $l_{C}$, while the electric field, $E_{3}$, is constant in the poled direction, namely $E_{3}=U / l_{C}$ (where $U$ is the drive voltage).

(5) Within the magnetostrictive rod, consider the magnetic field intensity $H_{1}=H_{2}=0$, while magnetic 


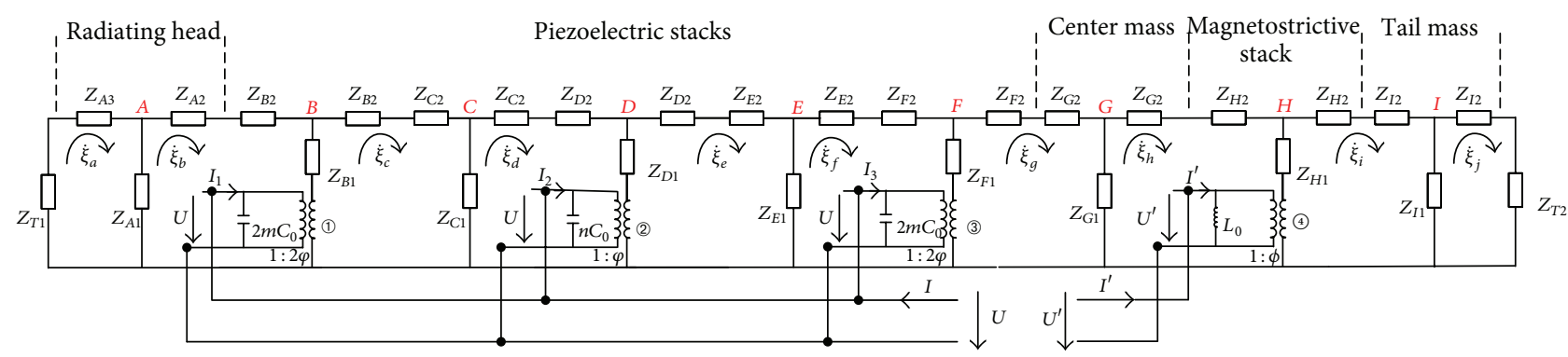

FIGURE 2: Equivalent circuit of the Hybrid transducer in water.

field intensity, $H_{3}$, is constant. Ampere's circuital law gives the relationship as $\oint_{l} H_{3} d l=\sum I$ (where $I$ is the drive current).

Based on the above assumptions, derived from the piezoelectric equation, piezomagnetic equation, geometric equation, and kinetic equation, the equivalent circuit will be obtained. Figure 2 illustrates the circuit representation, where every variable represents the meaning as follows:

$Z$ is the equivalent impedance labeled with the different subscripts such as " $A 1$ " and " $B 2$ " for the certain component of the Hybrid transducer.

$\dot{\xi}$ is the equivalent current labeled with the different subscripts for the certain loop in the circuit.

$C_{0}=\left(\varepsilon_{33}^{S} \cdot A_{C}\right) / l_{C}$ is the clamped capacitance (where $\varepsilon_{33}^{S}$ is the clamped dielectric constant), and $m, n$ are, respectively, the amounts of piezoelectric discs in the different stacks of the piezoelectric section.

$\varphi=A_{C} / l_{C} \cdot d_{33} / s_{33}^{E}$ is the electromechanical transformer of turns ratio for the piezoelectric section (where $d_{33}$ is the piezoelectric coefficient and $s_{33}^{E}$ is the short-circuit compliance). What should be paid attention to is the different values in the different places. Figure 2 shows that $1: \varphi$ is in the place of (2), while $1: 2 \varphi$ is in the place of (1) and (3). The essence of the above difference lies in the zigzag configuration. $1: \varphi$ will appear in the place where just one piezoelectric stack exists, while $1: 2 \varphi$ should be in the place where two piezoelectric stacks are parallel.

$L_{0}=N^{2} \mu_{33}^{S} A_{T} / L_{T}$ is the clamped inductance (where $N$ is the number of turns and $\mu_{33}^{S}$ is the clamped permeability coefficient).

$\phi=\widetilde{d}_{33} / j \omega N \mu_{33}^{S} s_{33}^{H}$ is the electromechanical transformer of turns ratio for the magnetostrictive section (where $\widetilde{d}_{33}$ is the piezomagnetic coefficient and $s_{33}^{H}$ is the compliance at constant magnetic field).

Specifically, every equivalent impedance in the equivalent circuit can be written as follows:

$$
\begin{aligned}
Z_{A 1}= & -j \cdot \frac{\sqrt{Z_{R 1} Z_{R 2}}}{\sin k_{R} L_{R}} \\
Z_{A 2}=-j & \cdot \frac{Z_{R 1}}{k_{R} L_{R}}\left(\sqrt{\frac{A_{R 2}}{A_{R 1}}}-1\right)-j \cdot \frac{Z_{R 1}}{\operatorname{tg} k_{R} L_{R}}+j \\
& \cdot \frac{\sqrt{Z_{R 1} Z_{R 2}}}{\sin k_{R} L_{R}},
\end{aligned}
$$

$$
\begin{aligned}
& Z_{A 3}=-j \cdot \frac{Z_{R 2}}{k_{R} L_{R}}\left(\sqrt{\frac{A_{R 1}}{A_{R 2}}}-1\right)-j \cdot \frac{Z_{R 2}}{\operatorname{tg} k_{R} L_{R}}+j \\
& \cdot \frac{\sqrt{Z_{R 1} Z_{R 2}}}{\sin k_{R} L_{R}} \\
& Z_{B 1}=-j \frac{2 Z_{C}^{E}}{\sin m k_{C} l_{C}}, \\
& Z_{B 2}=2 \cdot j Z_{C}^{E} \operatorname{tg} \frac{m k_{C} l_{C}}{2}, \\
& Z_{C 1}=-j \cdot \frac{Z_{V}}{\sin k_{V} L_{V}}, \\
& Z_{C 2}=j \cdot Z_{V} \cdot \operatorname{tg} \frac{k_{V} L_{V}}{2}, \\
& Z_{D 1}=-j \frac{Z_{C}^{E}}{\sin n k_{C} l_{C}} \\
& Z_{D 2}=j Z_{C}^{E} \operatorname{tg} \frac{n k_{C} l_{C}}{2}, \\
& Z_{E 1}=-j \cdot \frac{Z_{V}}{\sin k_{V} L_{V}}, \\
& Z_{E 2}=j \cdot Z_{V} \cdot \operatorname{tg} \frac{k_{V} L_{V}}{2}, \\
& Z_{F 1}=-j \frac{2 Z_{C}^{E}}{\sin m k_{C} l_{C}}, \\
& Z_{F 2}=2 \cdot j Z_{C}^{E} \operatorname{tg} \frac{m k_{C} l_{C}}{2}, \\
& Z_{G 1}=-j \cdot \frac{Z_{M}}{\sin k_{M} L_{M}}, \\
& Z_{\mathrm{G} 2}=j \cdot Z_{M} \cdot \operatorname{tg} \frac{k_{M} L_{M}}{2}, \\
& Z_{H 1}=-j \cdot \frac{Z_{T}^{H}}{\sin k_{T} L_{T}}+j \omega L_{0} \phi^{2}, \\
& Z_{H 2}=j \cdot Z_{T}^{H} \cdot \operatorname{tg} \frac{k_{T} L_{T}}{2},
\end{aligned}
$$




$$
\begin{aligned}
& Z_{I 1}=-j \cdot \frac{Z_{\mathrm{MM}}}{\sin k_{\mathrm{MM}} L_{\mathrm{MM}}}, \\
& Z_{I 2}=j \cdot Z_{\mathrm{MM}} \cdot \operatorname{tg} \frac{k_{\mathrm{MM}} L_{\mathrm{MM}}}{2},
\end{aligned}
$$

where the mechanical impedance can be written as the following representations:

$$
\begin{aligned}
Z_{R 1} & =\rho_{R} c_{R} A_{R 1}, \\
Z_{R 2} & =\rho_{R} c_{R} A_{R 2}, \\
Z_{C}^{E} & =\rho_{C} \cdot c_{C}^{E} \cdot A_{C}, \\
Z_{V} & =\rho_{V} \cdot c_{V} \cdot A_{V}, \\
Z_{M} & =\rho_{M} \cdot c_{M} \cdot A_{M}, \\
Z_{T}^{H} & =\rho_{T} \cdot c_{T}^{H} \cdot A_{T}, \\
Z_{\mathrm{MM}} & =\rho_{\mathrm{MM}} \cdot c_{\mathrm{MM}} \cdot A_{\mathrm{MM}},
\end{aligned}
$$

where $\rho, c, k, L, A$ are the density, sound velocity, wavenumber, length of certain component, and area of certain cross section, respectively. $A_{R 1}$ specially is the radiating surface area of the head, and $A_{R 2}$ is its laryngeal area. In the above representations, the subscript " $R$ " stands for the radiating head, " $C$ " stands for the piezoelectric ceramic, " $V$ " stands for the beam, " $M$ " stands for the center mass, "MM" stands for the magnetostrictive material, and " $T$ " stands for the tail mass.

In addition, $Z_{T 1}$ and $Z_{T 2}$ are the radiating impedance of the head and tail mass. Considering the low frequency, the following representation will be obtained [14]:

$$
Z_{T}=\rho_{W} \mathcal{c}_{W} A \cdot\left(\frac{k_{W} a}{2}\right)^{2}+j \rho_{W} c_{W} A \cdot \frac{2}{\pi}\left(k_{W} a\right),
$$

where the subscript " $W$ " stands for the water medium and " $a$ " is the radius of the radiating surface.

According to Figure 2, the relationships between voltages and currents can be obtained on the basis of Kirchhoff's voltage law. The materials and sizes of the main components are listed in Table 1. As a recommendation, it is an applicable way to use the matrix operation to solve the above problem. Consider

$$
[\mathbf{Z}] \cdot[\dot{\xi}]=[\mathbf{U}]
$$

where $[\mathbf{Z}],[\dot{\xi}]$, and $[\mathbf{U}]$ are the matrix representations about equivalent impedances, currents, and voltages.

It is obvious that the Hybrid transducer can be driven through 2 different ways, the piezoelectric stack with voltage excited and the magnetostrictive one with current excited. When the transducer is only driven by the voltage $U$ in the piezoelectric section while magnetostrictive section is open circuit, the admittance curves (including the conductance and susceptance) in water will be obtained, which is illustrated in Figure 3; on the other hand, if the transducer is
TABLE 1: The materials and sizes of the Hybrid transducer's main components.

\begin{tabular}{lll}
\hline Components & Materials & $\begin{array}{l}\text { Structure parameters } \\
(\mathrm{mm})\end{array}$ \\
\hline Head mass & Aluminum alloy & $\begin{array}{l}\text { Radiating surface } \\
\text { diameter } \Phi 70 \\
\text { Laryngeal diameter } \Phi 54 \\
\text { Length } L_{R}=20\end{array}$ \\
\hline Piezoelectric stack & P-41 & $\begin{array}{l}\text { Diameter } \Phi 13 \\
\text { Thickness } l_{C}=4 \\
\text { Amounts of piezoelectric } \\
\text { discs } m=8 \\
n=6\end{array}$ \\
\hline Beam & Steel & Thickness $L_{V}=5$ \\
\hline Center mass & Steel & $\begin{array}{l}\text { Diameter } \Phi 52 \\
\text { Length } L_{M}=20\end{array}$ \\
\hline Magnetostrictive rod & Terfenol_D & $\begin{array}{l}\text { Diameter } \Phi 20 \\
\text { Length } L_{T}=80\end{array}$ \\
\hline Tail mass & Steel & $\begin{array}{l}\text { Diameter } \Phi 54 \\
\text { Length } L_{M M}=55\end{array}$ \\
\hline
\end{tabular}

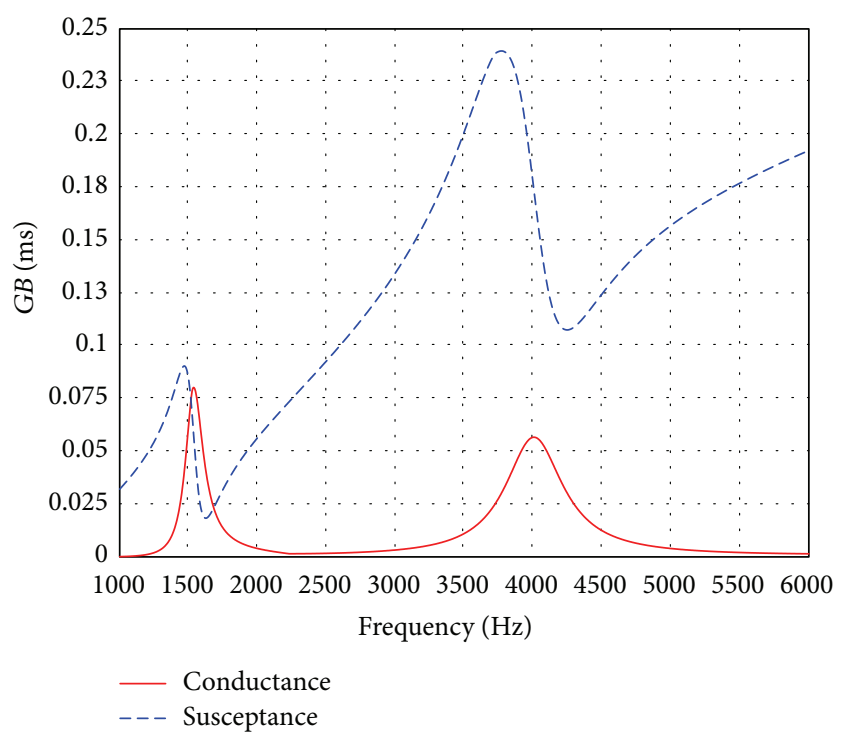

FIGURE 3: Admittance curves when only voltage drives in piezoelectric section (in water, obtained from equivalent circuit model).

only driven by the current $I^{\prime}$ in the magnetostrictive section while piezoelectric section is open circuit, the impedance curves (including the resistance and reactance) in water will be obtained, which is illustrated in Figure 4. All the curves show that the Hybrid transducer has 2 distinguishable resonances. One is $1.55 \mathrm{kHz}$ which is controlled by piezoelectric section and the other is $4.05 \mathrm{kHz}$ which is controlled by magnetostrictive section.

\section{Finite Element Model of the Hybrid Transducer}

The finite element method (FEM) is one of the best effective ways to design underwater transducer [15], whether its 


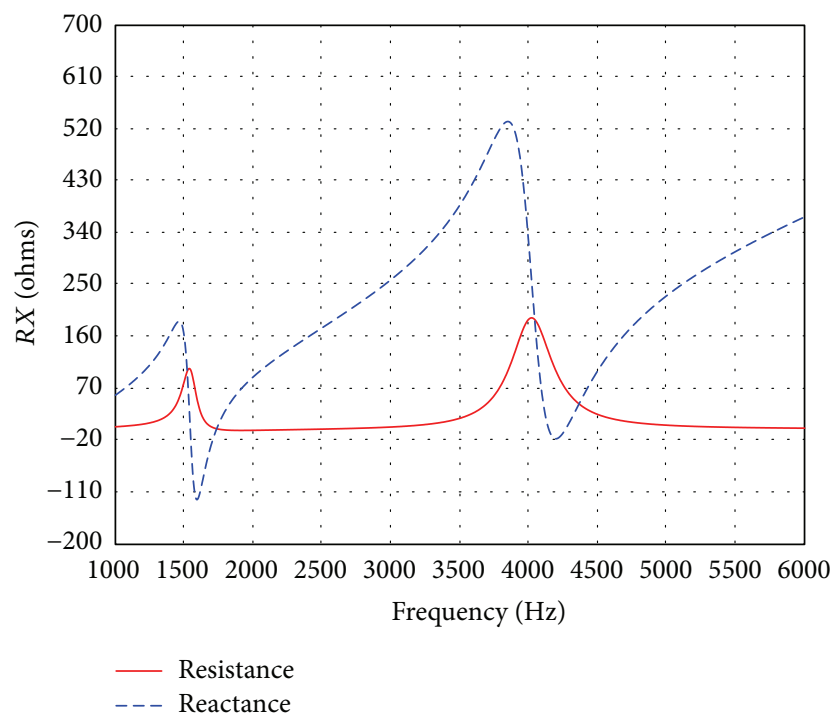

FIGURE 4: Impedance curves when only current drives in magnetostrictive section (in water, obtained from equivalent circuit model).

active material is piezoelectric or magnetostrictive. It is a numerical method by discretizing the whole system into finite elements. The program implements equations which govern the behavior of these elements and solves them all and then creates a comprehensive explanation of how the system acts as a whole. FEM is typically used for the design and optimization of a system which is too complex due to their configuration, scale, or governing equations to analyze by hand. Compared with the other theory models of underwater transducer, the large-scale assumption is not necessary for FEM. Anyway, FEM becomes an alternative consideration to model the transducer when its configuration is sophisticated or its boundary condition is complex.

During this analysis process, FEM need to handle the interaction between the structural and electric fields. When in water, the fluid-structure coupled-field is also necessary [16]. After the application of finite element discretization, the coupled finite element matrix equation [17] incorporated within the FEM software will be used to handle the above two coupled-field problems.

For the electric-structure coupled-field problem applied in piezoelectric analysis in piezoelectric section, FEM operates the control equation to solve the mechanical problem for linear material behavior in the following:

$$
[\mathbf{M}] \cdot[\ddot{\boldsymbol{\xi}}(t)]+[\mathbf{C}] \cdot[\dot{\boldsymbol{\xi}}(t)]+[\mathbf{K}] \cdot[\boldsymbol{\xi}(t)]=[\mathbf{F}(t)]
$$

where $[\boldsymbol{\xi}(t)]$ is vector of nodal displacements, $[\mathbf{M}]$ is mass matrix, $[\mathbf{C}]$ is damping matrix, $[\mathbf{K}]$ is stiffness matrix, and $[\mathbf{F}(t)]$ is nodal force vector, which determines the analysis types [18].

Combining with (5), after the discretization of finite element, the coupled finite element matrix equation about
TABLE 2: The analogous relationships between electric and magnetic variables.

\begin{tabular}{ll}
\hline Electric variables & Magnetic variables \\
\hline Electric field $E$ & Magnetic field $H$ \\
Electric displacement $D$ & Magnetic flux density $B$ \\
Voltage $U$ & Current $I$ \\
Short-circuit elastic modulus $c^{E}$ & Open circuit elastic modulus \\
& $c^{H}$ \\
Piezoelectric stress constant $e$ & Piezomagnetic strain constant \\
& $\tilde{e}$ \\
Clamped dielectric constant $\varepsilon^{S}$ & $\begin{array}{l}\text { Clamped permeability } \\
\text { constant } u^{S}\end{array}$ \\
\hline
\end{tabular}

structural and electrical field for one element model is given as follows:

$$
\begin{aligned}
& {\left[\begin{array}{ll}
\mathbf{M} & 0 \\
\mathbf{0} & \mathbf{0}
\end{array}\right] \cdot\left[\begin{array}{c}
\ddot{\boldsymbol{\xi}} \\
\ddot{\mathbf{U}}
\end{array}\right]+\left[\begin{array}{ll}
\mathbf{C} & \mathbf{0} \\
\mathbf{0} & \mathbf{0}
\end{array}\right] \cdot\left[\begin{array}{c}
\dot{\xi} \\
\dot{\mathbf{U}}
\end{array}\right]+\left[\begin{array}{cc}
\mathbf{K} & -\mathbf{K}^{Z} \\
\mathbf{K}^{Z} & \mathbf{K}^{d}
\end{array}\right] \cdot\left[\begin{array}{c}
\xi \\
\mathbf{U}
\end{array}\right]} \\
& =\left[\begin{array}{l}
\mathbf{F} \\
\mathbf{q}
\end{array}\right],
\end{aligned}
$$

where $[\mathbf{U}]$ is vector of nodal electrical potential, $\left[\mathbf{K}^{Z}\right]$ is piezoelectric coupling matrix, $\left[\mathbf{K}^{d}\right]$ is dielectric conductivity matrix, and $[\mathbf{q}]$ is electrical load vector.

Likewise, for the electric-structure coupled-field problem applied in piezomagnetic analysis in piezoelectric section, FEM still operates the same control equation like (5) and (6) for its linear behavior, but it is more complex. The reason lies in the magnetic-acoustic coupled-field problem in piezomagnetic analysis essentially. So what we need to do is to find the analogies between piezoelectric and piezomagnetic analysis, and then FEM can handle the piezomagnetic analysis by means of electric-structure coupled-field problem in piezoelectric analysis only with the replacements of variables [19]. The analogies of relevant variables are listed in Table 2. Using these analogies, the coupled finite element matrix equation for piezomagnetic analysis can be written as follows:

$$
\begin{aligned}
& {\left[\begin{array}{ll}
\mathbf{M} & \mathbf{0} \\
\mathbf{0} & \mathbf{0}
\end{array}\right] \cdot\left[\begin{array}{c}
\ddot{\boldsymbol{\xi}} \\
\ddot{\Gamma}
\end{array}\right]+\left[\begin{array}{ll}
\mathbf{C} & \mathbf{0} \\
\mathbf{0} & \mathbf{0}
\end{array}\right] \cdot\left[\begin{array}{c}
\dot{\boldsymbol{\xi}} \\
\dot{\boldsymbol{\Gamma}}
\end{array}\right]+\left[\begin{array}{cc}
\mathbf{K} & -\mathbf{K}^{Z} \\
\mathbf{K}^{Z} & \mathbf{K}^{d}
\end{array}\right] \cdot\left[\begin{array}{l}
\boldsymbol{\xi} \\
\mathbf{\Gamma}
\end{array}\right]} \\
& =\left[\begin{array}{l}
\mathbf{F} \\
\boldsymbol{\Phi}
\end{array}\right],
\end{aligned}
$$

where $[\boldsymbol{\Gamma}]$ is the ampere-turns around magnetostrictive rod and $[\Phi]$ is the magnetic flux.

Considering the analogies in Table 2, when the transducer is excited by current, $I$, the voltage, $U$, generated in exciting coils is $U=\partial \Phi / \partial t$, and the impedance of the transducer can be written as follows:

$$
Z=Z_{0}+\frac{\partial \Phi / \partial t}{I}
$$

where $Z_{0}$ is the clamped impedance. 


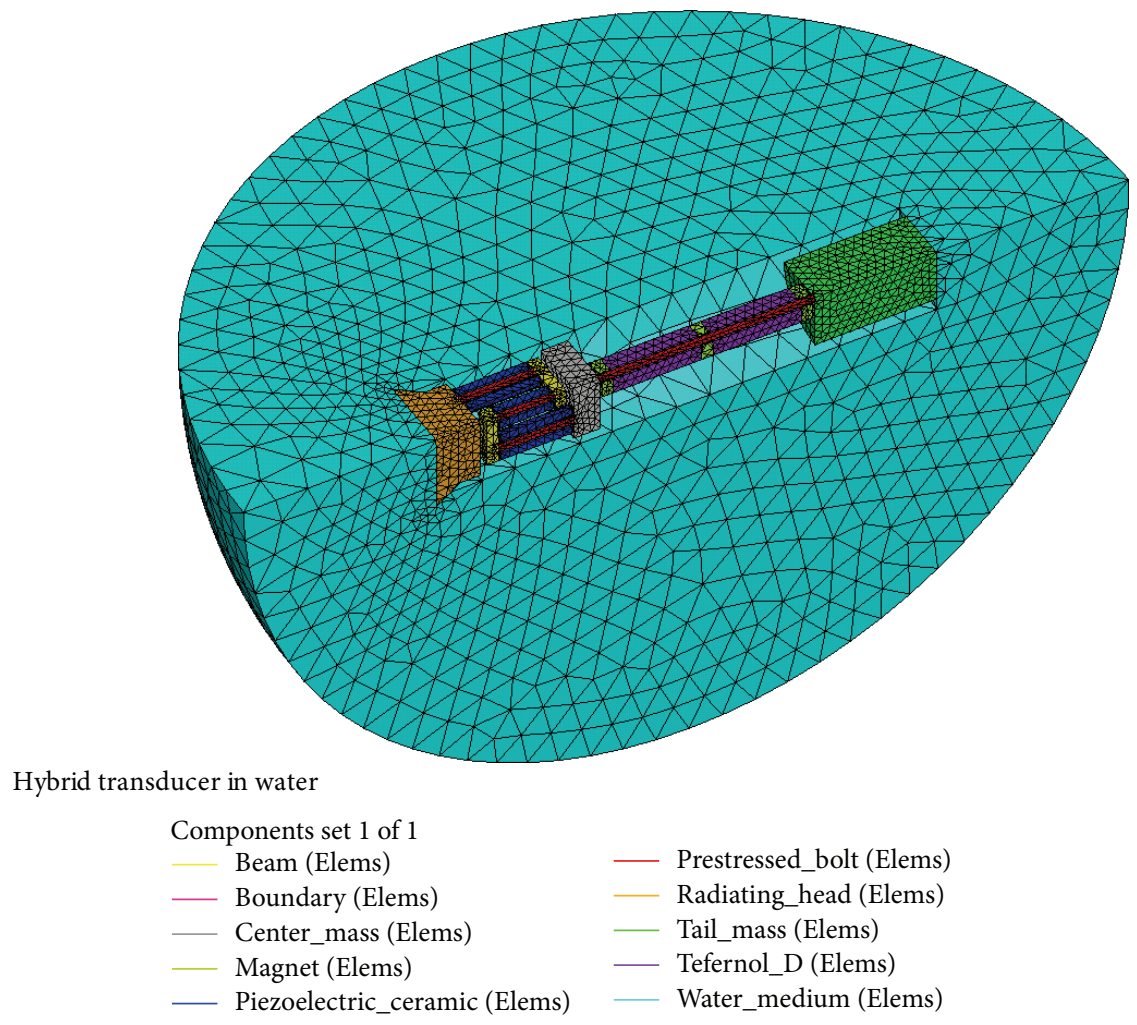

FIGURE 5: Finite element model of the Hybrid transducer in water (including 20701 nodes and 29496 elements).

It should be noted that the linear behaviour of the magnetostrictive material is considered in the above piezomagnetic analysis because we think its vibration evoked by the small electrical signal should be within its linear range. The analogies in Table 2 are not suitable for the nonlinear research of the magnetostrictive material.

For the fluid-structure coupled-field problem in underwater acoustic analysis, FEM operates the control equation in the following [18]:

$$
\begin{gathered}
{\left[\begin{array}{cc}
\mathbf{M} & \mathbf{0} \\
\mathbf{M}^{\mathrm{fs}} & \mathbf{M}^{f}
\end{array}\right] \cdot\left[\begin{array}{c}
\ddot{\boldsymbol{\xi}} \\
\ddot{\mathbf{P}}
\end{array}\right]+\left[\begin{array}{cc}
\mathbf{C} & \mathbf{0} \\
\mathbf{0} & \mathbf{C}^{f}
\end{array}\right] \cdot\left[\begin{array}{c}
\dot{\boldsymbol{\xi}} \\
\dot{\mathbf{P}}
\end{array}\right]+\left[\begin{array}{cc}
\mathbf{K} & \mathbf{K}^{\mathrm{fs}} \\
\mathbf{0} & \mathbf{K}^{f}
\end{array}\right]} \\
\cdot\left[\begin{array}{l}
\boldsymbol{\xi} \\
\mathbf{P}
\end{array}\right]=\left[\begin{array}{l}
\mathbf{F} \\
\mathbf{0}
\end{array}\right],
\end{gathered}
$$

where $\mathbf{P}$ is the acoustic pressure and $\left[\mathbf{M}^{\mathrm{fs}}\right]$ is the coupled mass matrix of the fluid-structure interface, in which the superscript " $f s$ " stands for fluid-structure interface; for $\left[\mathbf{M}^{f}\right]$, $\left[\mathbf{C}^{f}\right]$, and $\left[\mathbf{K}^{f}\right]$, the superscript " $f$ " stands for water medium.

Once the above control equations are obtained, the suitable element type of FEM should be confirmed, and then we can build the model. For our Hybrid transducer, the following element type listed in Table 3 will be used.

According to the definitions in Tables 1 and 3, a FEM model illustrated in Figure 5 has been constructed in the multiphysics or mechanical products of ANSYS, which is one of the most famous commercial finite element software programs. Considering a simple model and the computation speed, a whole model is not recommended. Figure 5 illustrates a $1 / 4$ symmetrical finite element model. The necessary degrees of freedom (DOF) have been defined by selecting appropriate element type and setting the right option (see Table 3). And Table 1 gives the materials and size of the main components. When modeling, we should input the right material properties to define the polarization direction of the piezoelectric stacks or the bias magnetic field direction of the magnetostrictive rod aligned along the axis. A point to remember is that the input order must follow the ANSYS standard [17], not the IEEE standard [20]. The conversion between the two standards [21] is necessary in ANSYS analysis because the input order follows the IEEE standard in most published references.

During the analysis, the suitable analysis types should be set up to achieve our interested analysis objective. Generally, the possible analysis types are static, modal, prestressed modal, harmonic, prestressed harmonic, and transient. Static analysis can be used to determine the stresses and strains when the transducer is prestressed. Modal analysis can be used to determine the operating frequencies and mode shapes of a transducer, also applied on a prestressed structure. Harmonic response analysis gives us the graph of some responses versus frequency; that is, admittance, bandwidth, efficiency, and even acoustic radiating field can be obtained. A whole prediction about underwater transducer, including the mechanical, electrical, and acoustical, will be achieved through the above analysis. 
TABLE 3: Material properties and description of Hybrid transducer.

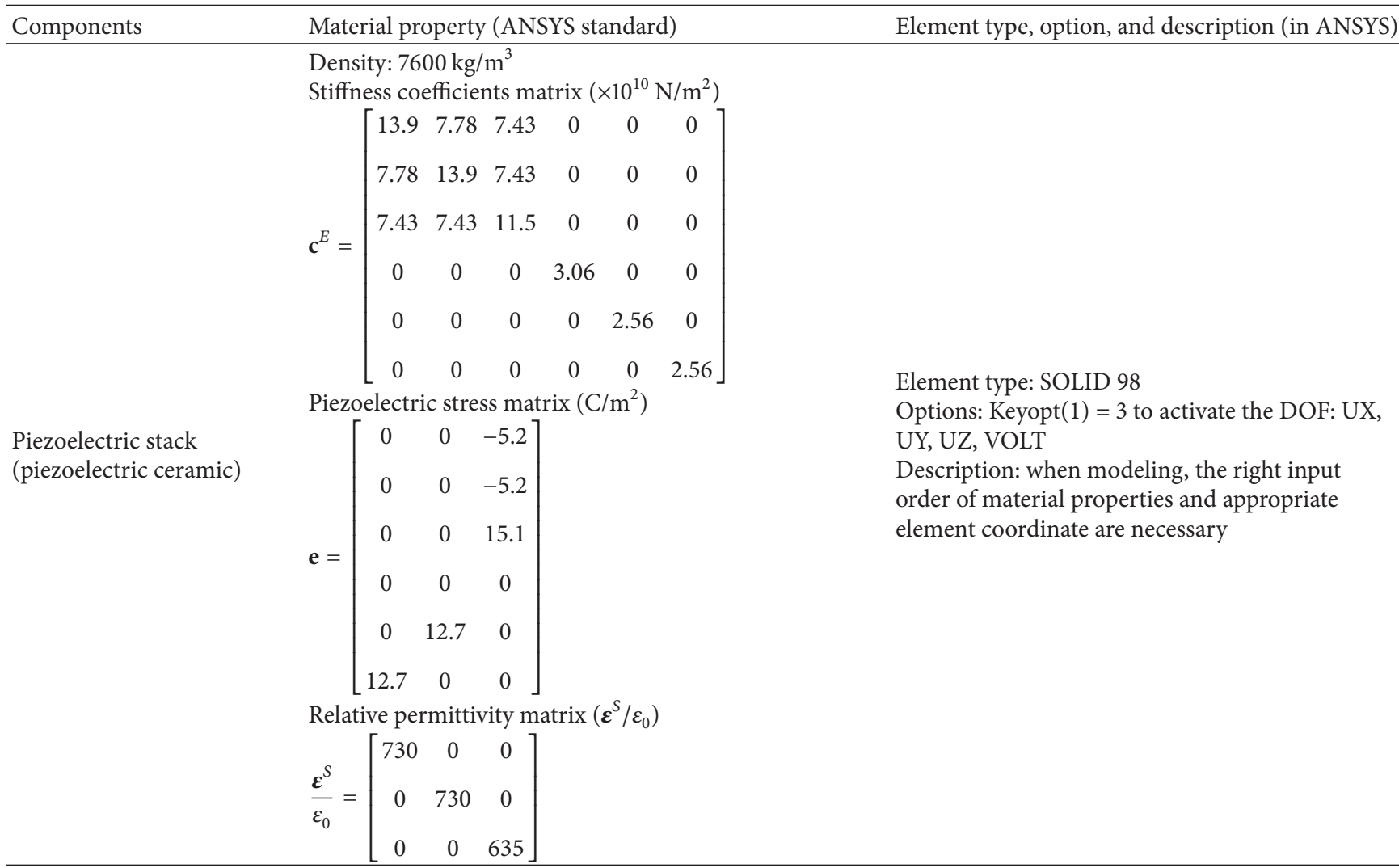

Density: $9250 \mathrm{~kg} / \mathrm{m}^{3}$

Compliance coefficients matrix $\left(\times 10^{-11} \mathrm{~m}^{2} / \mathrm{N}\right)$

Magnetostrictive stack (Tefernol_D) [13]

$$
\mathbf{s}^{H}=\left[\begin{array}{cccccc}
4.4 & -1.1 & -1.65 & 0 & 0 & 0 \\
-1.1 & 4.4 & -1.65 & 0 & 0 & 0 \\
-1.65 & -1.65 & 3.8 & 0 & 0 & 0 \\
0 & 0 & 0 & 11 & 0 & 0 \\
0 & 0 & 0 & 0 & 24 & 0 \\
0 & 0 & 0 & 0 & 0 & 24
\end{array}\right]
$$

Piezomagnetic charge matrix $\left(\times 10^{-9} \mathrm{~m} / \mathrm{A}\right)$

$$
\widetilde{\mathbf{d}}=\left[\begin{array}{ccc}
0 & 0 & -4.3 \\
0 & 0 & -4.3 \\
0 & 0 & 8.5 \\
0 & 0 & 0 \\
0 & 16.5 & 0 \\
16.5 & 0 & 0
\end{array}\right]
$$

Relative permeability matrix $\left(\boldsymbol{\mu}^{T} / \mu_{0}\right)$

$$
\frac{\boldsymbol{\mu}^{T}}{\mu_{0}}=\left[\begin{array}{ccc}
8.1 & 0 & 0 \\
0 & 8.1 & 0 \\
0 & 0 & 3.0
\end{array}\right]
$$

Density: $2700 \mathrm{~kg} / \mathrm{m}^{3}$

Radiating head (aluminum alloy)
Young's modulus: $7.1 \times 10^{10} \mathrm{~N} / \mathrm{m}^{2}$ Poisson's ratio: 0.33
Element type: SOLID 98

Options: $\operatorname{Keyopt}(1)=3$ to activate the DOF: UX, UY, UZ, VOLT

Description: when modeling, the right input order of material properties and appropriate element coordinate are necessary
Element type: SOLID 98

Options: $\operatorname{Keyopt}(1)=2$ to activate the DOF: UX, UY, UZ 
TABLE 3: Continued.

\begin{tabular}{lll}
\hline Components & Material property (ANSYS standard) & Element type, option, and description (in ANSYS) \\
\hline $\begin{array}{l}\text { Center mass } \\
\text { Tail mass (steel) }\end{array}$ & $\begin{array}{l}\text { Density: } 7840 \mathrm{~kg} / \mathrm{m}^{3} \\
\text { Young's modulus: } 2.16 \times 10^{11} \mathrm{~N} / \mathrm{m}^{2} \\
\text { Poisson's ratio: } 0.28\end{array}$ & $\begin{array}{l}\text { Element type: SOLID 98 } \\
\text { Options: Keyopt(1) }=2 \text { to activate the DOF: UX, } \\
\text { UY, UZ }\end{array}$ \\
\hline Water medium (water) & $\begin{array}{l}\text { Density: } 1000 \mathrm{~kg} / \mathrm{m}^{3} \\
\text { Velocity of sound: } 1481 \mathrm{~m} / \mathrm{s}\end{array}$ & $\begin{array}{l}\text { Element type: FLUID } 30 \\
\text { Options: Keyopt(2) }=0 \text { to activate the DOF: UX, } \\
\text { UY, UZ and PRES }\end{array}$ \\
\hline \multirow{3}{*}{ Boundary } & Keyopt $(1)=1$ to active the DOF: PRES \\
& $\begin{array}{l}\text { Density: } 1000 \mathrm{~kg} / \mathrm{m}^{3} \\
\text { Velocity of sound: } 1481 \mathrm{~m} / \mathrm{s}\end{array}$ & $\begin{array}{l}\text { Element type: FLUID 130 } \\
\text { Real constant: RAD will be specified as the radius } \\
\text { of the spherical boundary of the finite domain }\end{array}$ \\
\hline
\end{tabular}

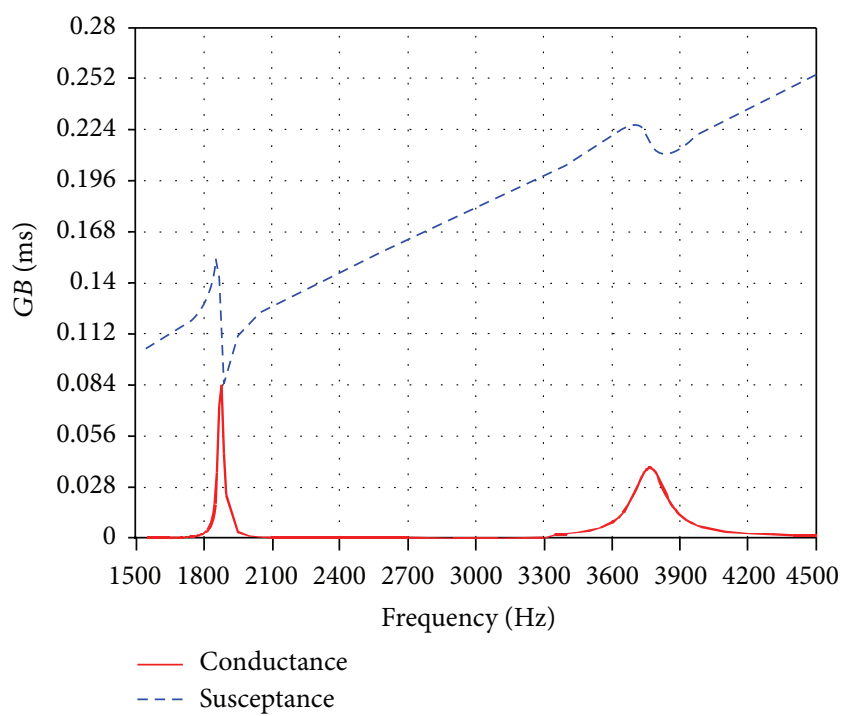

FIGURE 6: Admittance curves when only voltage drives in piezoelectric section (in water, obtained from finite element model).

Here, the impedance performances of Hybrid transducer in water have been presented. Figure 6 shows the admittance curves obtained from the single voltage drive, and Figure 7 shows the impedance curves obtained from the single current drive. The two main resonances of the Hybrid transducer are $1.88 \mathrm{kHz}$ and $3.77 \mathrm{kHz}$, respectively.

\section{Test and Discussion}

A Hybrid transducer prototype has been manufactured according to the above design. The details about the materials and sizes are listed in Tables 1 and 3. Figure 8 illustrates the prototype. Its main body has an external diameter of $54 \mathrm{~mm}$. The whole prototype is $235 \mathrm{~mm}$ long and weighs $2.61 \mathrm{~kg}$ (without house and cable).

Figure 9 is the admittance curves obtained from the single voltage drive, and Figure 10 is the impedance curves obtained from the single current drive. Both are obtained from the precision impedance analyzer Agilent 4294A. All the curves show that the prototype has 2 resonances, namely, $1.82 \mathrm{kHz}$ and $3.76 \mathrm{kHz}$.

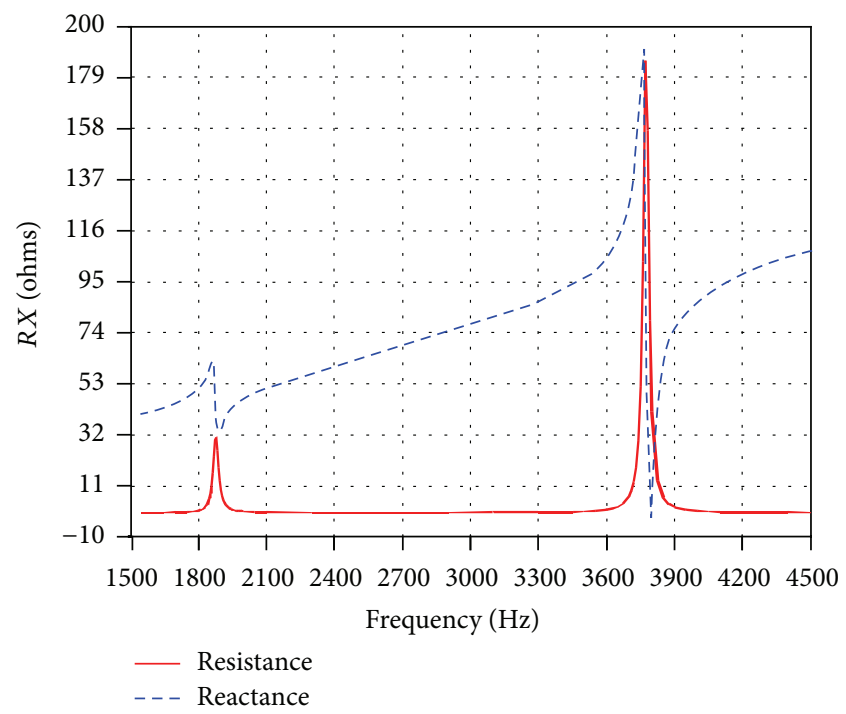

FIGURE 7: Impedance curves when only current drives in magnetostrictive section (in water, obtained from finite element model).

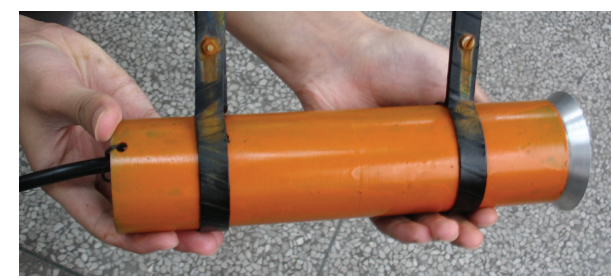

FIgURE 8: The Hybrid transducer prototype (including house).

Since the drive way such as voltage drive or current drive is alternative, it is hard for our Hybrid transducer to estimate its transmitting ability only through either the single transmitting voltage response (TVR) or the single transmitting current response (TCR). Considering the convenience to compare with the other similar transducers, a test has been finished within the range of $1.5 \sim 5 \mathrm{kHz}$, and the max TVR is $124.5 \mathrm{~dB}$ at the frequency of $1.85 \mathrm{kHz}$ when only the voltage drives in the piezoelectric section while the max TCR is $185.4 \mathrm{~dB}$ at the frequency of $3.5 \mathrm{kHz}$ when only the current drives in the magnetostrictive section. It is certain that the number of turns of 682 makes important contribution to very 
TABLE 4: Comparison between MPT (NUMC) and Hybrid transducer (this paper).

\begin{tabular}{|c|c|c|}
\hline & MPT (NUMC) & Hybrid transducer (this paper) \\
\hline Length $(\mathrm{mm})$ & 406 & 235 \\
\hline Radiating surface $(\mathrm{mm})$ & Square, $190.5 \times 190.5$ & Circle, $\Phi 70$ \\
\hline \multicolumn{3}{|l|}{ Weight (kg) } \\
\hline Without house & 29.5 & 2.61 \\
\hline With house & 40 & 3.3 \\
\hline $\begin{array}{l}\text { Volume of piezoelectric } \\
\text { ceramic }\left(\times 10^{3} \mathrm{~mm}^{3}\right)\end{array}$ & Not known & 15.87 \\
\hline $\begin{array}{l}\text { Volume of magnetostrictive } \\
\text { material }\left(\times 10^{3} \mathrm{~mm}^{3}\right)\end{array}$ & Not known & 22.86 \\
\hline Bias magnetic field $(\mathrm{kA} / \mathrm{m})$ & 60 & 40 \\
\hline Number of turns $(N)$ & 300 & 682 \\
\hline \multicolumn{3}{|l|}{ Resonance frequency $(\mathrm{kHz})$} \\
\hline Low & 1.8 & 1.82 \\
\hline High & 3.5 & 3.76 \\
\hline $\begin{array}{l}\text { Max transmitting response } \\
(\mathrm{dB})\end{array}$ & $\begin{array}{c}152(\mathrm{TVR}) \\
\text { Voltage drives in both piezoelectric and } \\
\text { magnetostrictive sections }\end{array}$ & $\begin{array}{c}124.5 \text { (TVR) } \\
\text { Only voltage drives in the piezoelectric } \\
\text { section } \\
185.4 \text { (TCR) } \\
\text { Only current drives in the magnetostrictive } \\
\text { section } \\
\end{array}$ \\
\hline
\end{tabular}

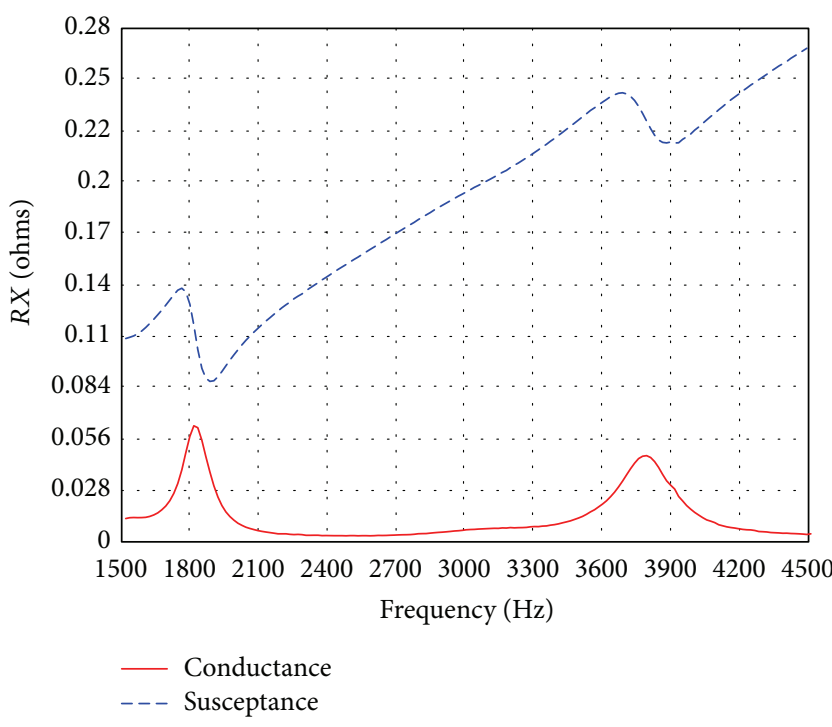

FIGURE 9: Admittance curves when only voltage drives in piezoelectric section (in water, obtained from Agilent 4294A).

high max TCR; the suitable bias magnetic field of $40 \mathrm{kA} / \mathrm{m}$ or so and the prestressing force of about $10 \mathrm{MPa}$ are also beneficial.

A comparison is illustrated in Table 4, where MPT is a Hybrid magnetostrictive/piezoelectric transducer developed at the Naval Undersea Warfare Center (NUWC) [22]. The two Hybrid transducers have the same operating frequency range. According to the comparison, our transducer prototype is $60 \%$ of the MPT in length, $9 \%$ of the MPT in weight, and

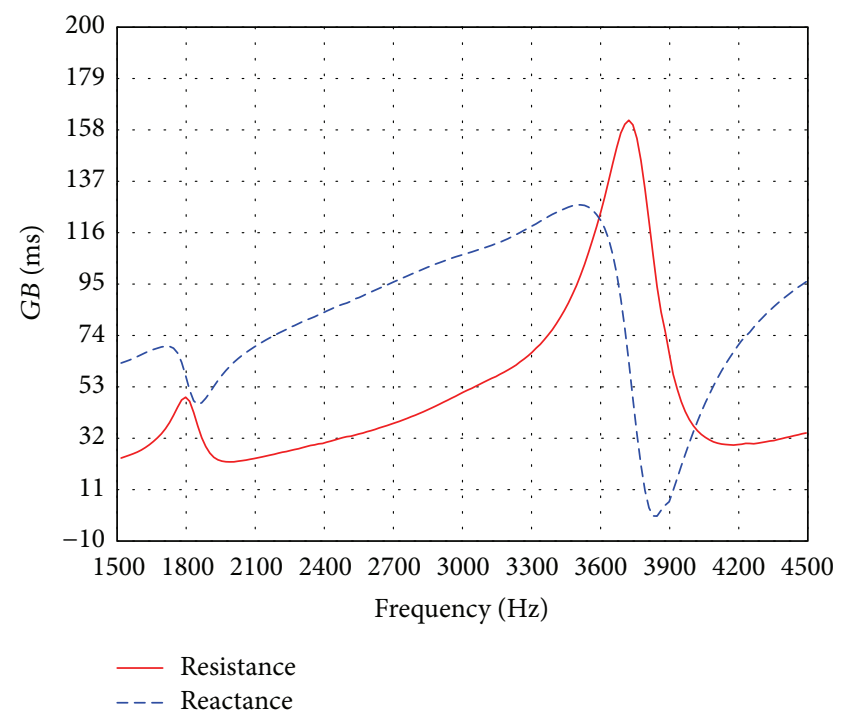

FIGURE 10: Impedance curves when only current drives in magnetostrictive section (in water, obtained from Agilent 4294A).

$10 \%$ of MPT in cross section. But the max transmitting sound level of our transducer is less than MPT's because the volume of their active material has distinct difference.

\section{Conclusions}

A novel Hybrid transducer is developed. Its performance has been predicted through the method of equivalent circuit model and finite element model. The analysis results and 
the test results show that the method of FEM is suitable to this type transducer, and its analysis result is believable. A transducer prototype according to the design has the main body external diameter of $54 \mathrm{~mm}$. The whole prototype is $235 \mathrm{~mm}$ long and weighs $2.61 \mathrm{~kg}$. It has the resonance at $1.82 \mathrm{kHz}$ and $3.76 \mathrm{kHz}$. It can be used effectively in the bandwidth of $1.5 \mathrm{kHz}$ to $5 \mathrm{kHz}$.

\section{Competing Interests}

The authors declare that they have no competing interests.

\section{Acknowledgments}

This project is supported by the Fundamental Research Funds for the Central Universities (3102014KYJD009) and the Funds for the National Key Laboratory (9140C230304140C23140).

\section{References}

[1] F. Massa, Sonar Transducers: A History, ST Special Report, Sea Technology, 1989.

[2] J.-N. Decarpigny, B. Hamonic, and O. B. Wilson Jr., "The design of low-frequency underwater acoustic projectors: present status and future trends," IEEE Journal of Oceanic Engineering, vol. 16, no. 1, pp. 107-122, 1991.

[3] S. C. Butler, “Triply resonant broadband transducers," in Proceedings of the MTS/IEEE (OCEANS '02), vol. 4, pp. 2334-2341, October 2002.

[4] W. Lu, Z. Y. Zhang, and Y. Lan, "Research of the new low frequency, broadband free-flooded piezoelectric underwater acoustic transducer," in Proceedings of the Joint Conference of the Symposium on Piezoelectricity, Acoustic Waves, and Device Applications (SPAWDA '09) and China Symposium on Frequency Control Technology, pp. 41-44, 2009.

[5] Y. Pan, X. Mo, Y. Chai, Y. Liu, and Z. Cui, "A new design on broadband flextensional transducer," Applied Acoustics, vol. 72, no. 11, pp. 836-840, 2011.

[6] C. H. Sherman and J. L. Butler, Transducers and Arrays for Underwater Sound, 1st edition, corrected 2nd printing, Springer, New York, NY, USA, 2007.

[7] S. C. Butler and J. F. Lindberg, "Hybrid magnetostrictive/piezoelectric Tonpilz transducer," Ferroelectrics, vol. 187, no. 1, pp. 163-174, 1996.

[8] J. L. Butler, A. L. Butler, and S. C. Butler, "Hybrid magnetostrictive/piezoelectric Tonpilz transducer," Journal of the Acoustical Society of America, vol. 94, no. 2, pp. 636-641, 1993.

[9] W. P. Mason, Physical Acoustics and the Properties of Solids, chapter 3, Van Nostrand, Jersey City, NJ, USA, 1958.

[10] H. A. C. Tilmans, "Equivalent circuit representation of electromechanical transducers: I. Lumped-parameter systems," Journal of Micromechanics and Microengineering, vol. 6, no. 1, pp. 157-176, 1996.

[11] H. A. C. Tilmans, "Equivalent circuit representation of electromechanical transducers: II. Distributed-parameter systems," Journal of Micromechanics and Microengineering, vol. 7, no. 4, pp. 285-309, 1997.

[12] F. H. Zhou, Underwater Transducers and Arrays, National Defence Industry Press, 1st edition, 1984.
[13] F. Claeyssen, N. Lhermet, and F. Barillot, "Giant dynamic strains in magnetostrictive actuators and transducers," in Proceedings of the ISAGMM, pp. 1-15, Guiyang, China, October 2006.

[14] J. H. Gu and X. Q. Ye, Fundamental of Underwater Acoustic, National Defence Industry Press, 1st edition, 1981.

[15] J. Mackerle, "Smart materials and structures-a finite element approach-an addendum: a bibliography (1997-2002)," Modelling and Simulation in Materials Science and Engineering, vol. 11, no. 5, pp. 707-712, 2003.

[16] ANSYS, ANSYS Fluids Analysis Guide, Release 9.0, ANSYS, Canonsburg, Pa, USA, 2004.

[17] ANSYS, ANSYS User's Manual, Release 10.0, ANSYS, Canonsburg, Pa, USA, 2006.

[18] ANSYS Inc, Coupled-Field Analysis Guide, Release 12.1, ANSYS Inc, 2009.

[19] X. P. Mo, Z. H. Qing, J. G. Liu, and Z. Cui, "Terfenol-D giant magnetostrictive transducer simulation by finite element method," Journal of Applied Acoustics, vol. 19, no. 4, pp. 5-8, 2000.

[20] ANSI/IEEE, "An American National Standard IEEE standard on piezoelectricity," ANSI/IEEE Std 176-1987, 1987.

[21] S. Imaoka, Conversion of Piezoelectric Material Data, Collaborative Solutions Inc, 1999.

[22] S. C. Bulter and F. A. Tito, "A broadband hybrid magnetostrictive/piezoelectric transducer array," in Proceedings of the Oceans 2000 MTS/IEEE Conference and Exhibition, vol. 3, pp. 14691475, Providence, RI, USA, September 2000. 


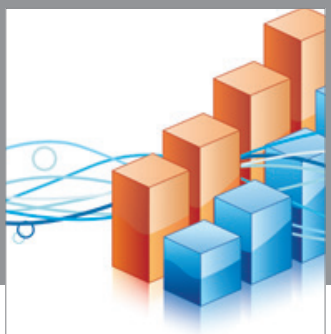

Advances in

Operations Research

vatem alat4

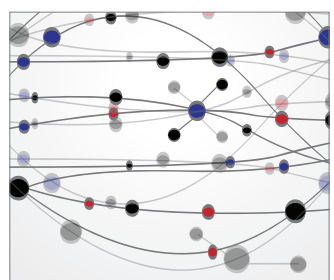

\section{The Scientific} World Journal
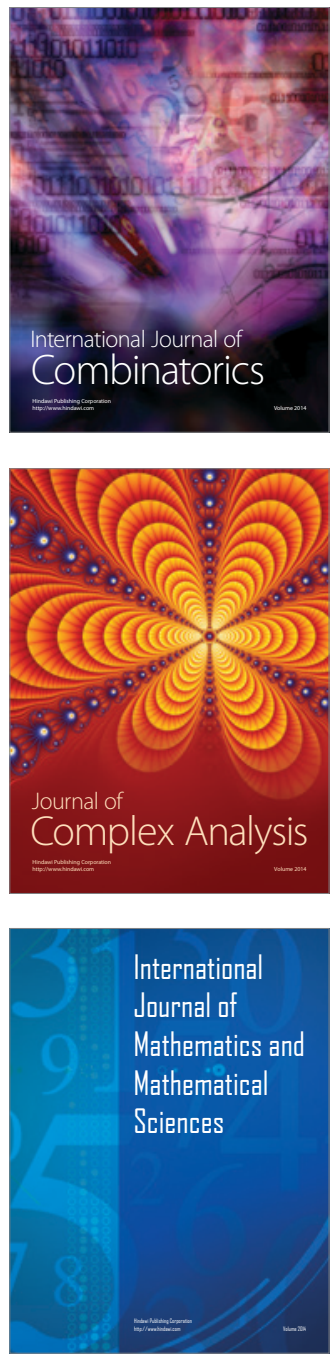
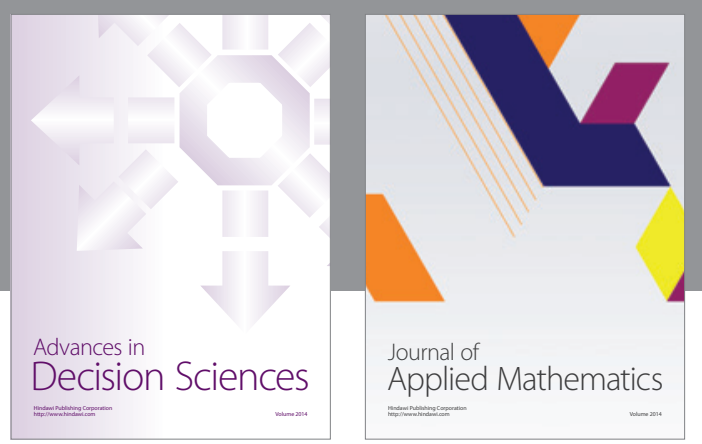

Algebra

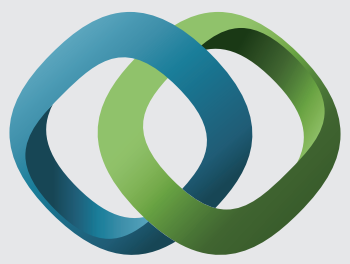

\section{Hindawi}

Submit your manuscripts at

http://www.hindawi.com
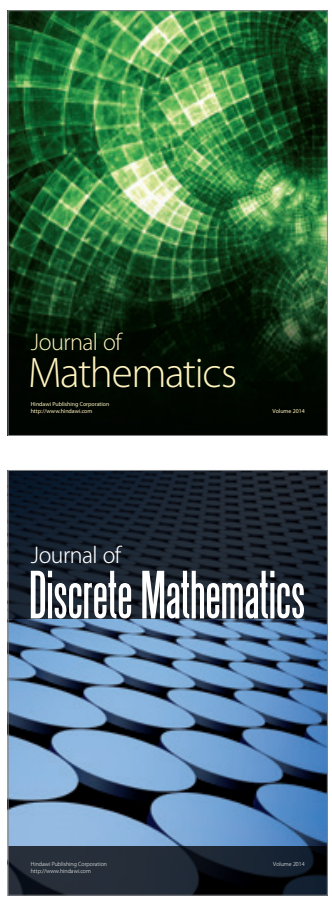

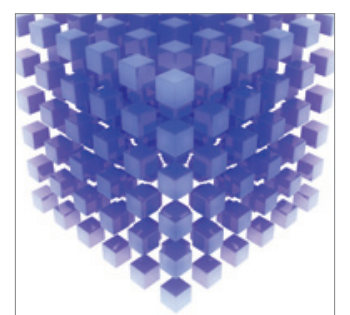

Mathematical Problems in Engineering
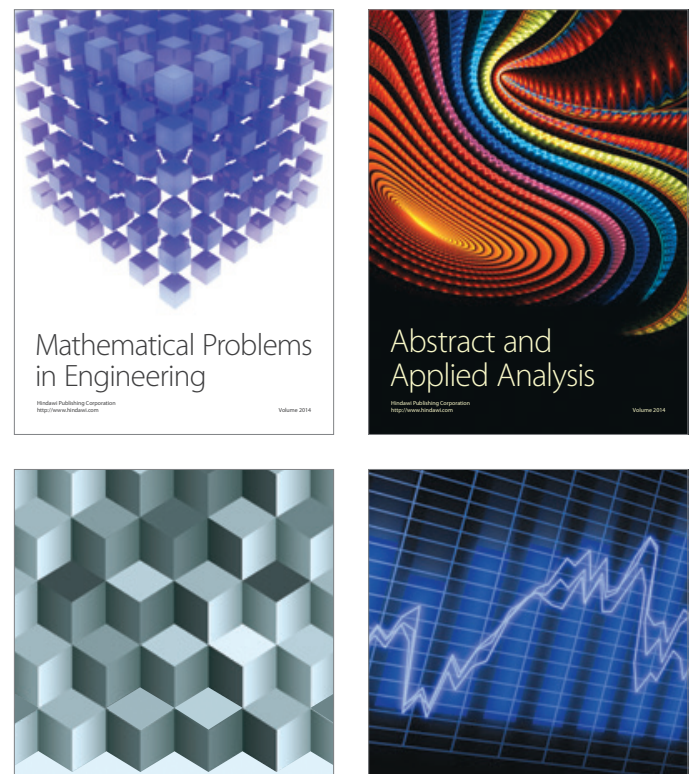

Journal of

Function Spaces

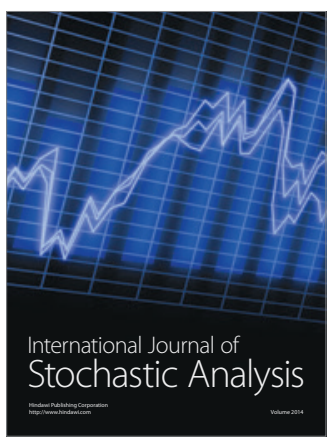

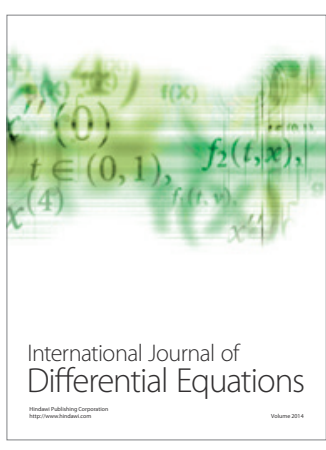
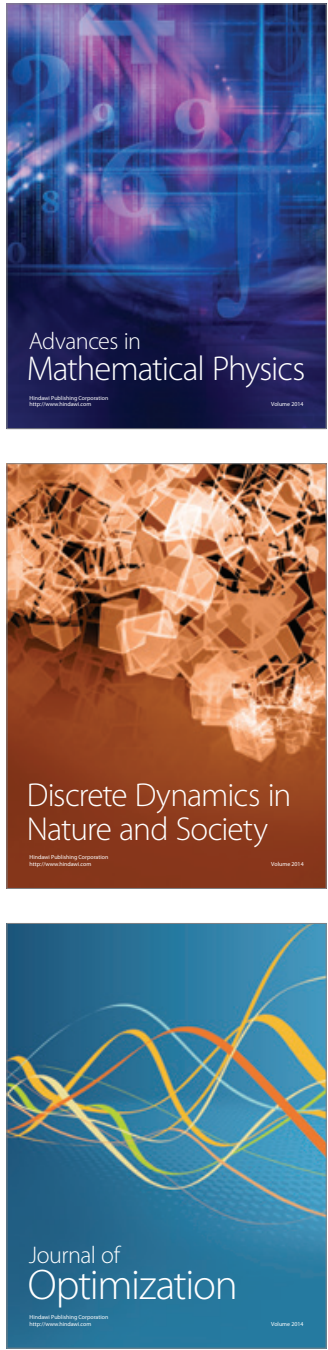